\title{
Self-IQ-Demodulation Based Compensation Scheme of Frequency-Dependent IQ Imbalance for Wideband Direct-Conversion Transmitters
}

\author{
Wei Li, Yue Zhang, Li-ke Huang, Jin Wang, John Cosmas, Carsten Maple, Jian Xiong
}

\begin{abstract}
A low cost frequency-dependent (FD) I/Q imbalance self-compensation scheme is investigated in this paper. The direct conversion transmitters are widely used in wireless systems. However, the unwanted image-frequencies and distortions are inevitably introduced into the direct conversion system. This problem is even severer in wideband systems. Therefore, the accurate estimation and compensation of $I / Q$ imbalance is crucial. The current compensation method is based on external instruments or internal feedback path which introduces additional impairments and is expensive. This paper proposes a low cost FD I/Q imbalance self-IQ-demodulation based compensation scheme without using external calibration instruments. First, the impairments of baseband and RF components are investigated. Further, I/Q imbalance model is developed. Then, the proposed two-step self-IQ-demodulation based compensation scheme is investigated. In the first step of the scheme, the local oscillator (LO) related I/Q impairments parameters are estimated. Then in the second step, the overall FD I/Q imbalance parameters are estimated by utilizing the transmitter LO. To realize this self-IQ-demodulation algorithm, this paper introduces minor modifications to the current power detector circuit. Afterwards, the estimated parameters are applied to the baseband equivalent compensator. This sophisticated algorithm guarantees low computation complexity and low cost. The compensation performance is evaluated in laboratory measurement.
\end{abstract}

Index Terms - direct conversion transmitters, IQ imbalance, image-frequency interference, automatic calibration, LO, RF

Wei $\mathrm{Li}$ is a $\mathrm{PhD}$ student at the Institute for Research in Applicable Computing, University of Bedfordshire, Luton, LU1 3JU, UK (e-mail: Wei.Li@beds.ac.uk)

Yue Zhang is a Reader in Signal Processing at the Department of Computer Science and Technology, University of Bedfordshrie, UK (e-mail: yue_zhang@ieee.org)

Li-Ke Huang is a technical expert and the Algorithms Group Leader at Aeroflex UK, (email: Li-ke.Huang@aeroflex.com)

Jin Wang is a a principal engineer at Aeroflex UK (e-mail: Jin.Wang (a)aeroflex.com )

John Cosmas, is a professor of Multimedia Systems in the School of Engineering and Design Brunel University, London, UB8 3PH, UK. (e-mail: John.Cosmas@Brunel.ac.uk)

Carsten Maple is a Professor of University Bedfordshire, Luton, LU1 3JU, UK (e-mail: Carsten.Maple@beds.ac.uk)

Jian Xiong is a Associate Professor at the Department of Electronic Engineering, Shanghai Jiao Tong University, China (e-mail: xjarrow@sjtu.edu.cn)

\section{INTRODUCTION}

$\mathrm{T}$ HE direct-conversion transmitters (DCT) are widely adopted in digital broadcasting systems such digital audio broadcasting (DAB), digital video broadcasting (DVB), digital terrestrial multimedia broadcasting (DTMB) because of the advantages of low-cost, simplicity and flexibility. The ideal digital complex signal is represented by inphase and quadrature signal in I and Q branch respectively. The baseband I and Q signal is then modulated by quadrature modulator which makes DCT easy to implement. However, some practical problems arise from the implementation of direct-conversion transmitters. These problems are mainly caused by non-idealities of the baseband and RF components, such as I/Q imbalance, clock jitter, power amplifier (PA) distortion and inter-modulation. The overall impact results in image-frequency interference, phase noise and high order frequency interference, which severely degrades the transmitter performance especially for the wideband multicarrier systems. Generally, the main impact of I/Q imbalance is the image-frequency interference and degradation of PA nonlinearity [1], [14-16]. In OFDM systems such as DVB, the image frequency component overlaps the desired signal on corresponding subcarriers, which decrease SNR of the desired signal. Hence, image rejection ratio (IRR) is a critical criterion for DCT.

There has been several work that has been reported in the literature. The work in [4] copes with combined effects of transmitter and receiver I/Q imbalance, whereas, the work in [1-3] focuses on transmitter I/Q imbalance and adopts low intermediate frequency (IF) down-conversion structure, in which the corrupted signal is down-converted to a low IF and sample with an analog-to-digital converter (ADC), which requires high speed ADC. I/Q demodulation and parameters estimation are computed by DSP. The advantage of this estimation structure is that $\mathrm{I} / \mathrm{Q}$ demodulation are performed in digital domain, which avoids the receiver $\mathrm{I} / \mathrm{Q}$ imbalance. [5]-[7] also investigated the low-cost diode-based I/Q imbalance estimation technique, which makes use of the power detector of transmitters and realizes self-calibration. However, the estimation is confined to frequency-independent I/Q imbalance due to the bandwidth limit of power detector. [1-3] studies frequency-independent $\mathrm{I} / \mathrm{Q}$ imbalance, which is more realistic for wideband systems such as DVB and LTE. [1] 
proposes second-order statistics-based approach and widely linear least-squares (WL-LS) approach which do not take into account of LO leakage. [2] estimates the I/Q imbalance parameters by cross-processing between I and $\mathrm{Q}$ signals. [3] proposes a specially designed pilot sequence, which estimates $\mathrm{I} / \mathrm{Q}$ imbalance and channel response jointly.

In this paper, a self-IQ-demodulation based compensation scheme of FD I/Q imbalance is studied and presented. Specially, the "time division" method and its corresponding circuit are implemented to realize self-IQ-demodulation. This compensation scheme consists of estimation and compensation stages. The estimation stage takes a two-step self-IQ-demodulation algorithm. In the first step, the amplitude LO impairments including DC offset is estimated using the power detector. The special training signal is designed with low computation complexity. Second, the self-I/Q-demodulation is implemented to estimate the frequency-dependent I/Q imbalance parameters. As the external I/Q demodulator has been omitted in this scheme, the transmitter LO is transmitted with the test signal using "time division" method for self-I/Q-demodulation. The LO related impairments parameters, which are estimated in step 1 are then used to compensate the self-I/Q-demodulation error caused by the inherent transmitter LO imbalance. Afterwards, the FD I/Q imbalance can be estimated. Furthermore, the estimation results are applied to the compensation stage. The circuit components in [5-7] are extended to wideband in this proposal to meet the requirement of self-I/Q-demodulation,.

The organization for the rest of the paper is as follows. The analysis and model of FD I/Q imbalance are presented in Section II. The new I/Q imbalance estimation scheme including the compensation technique is discussed in Section III. In Section IV, the simulation and laboratory experiments results are presented. The conclusions are drawn in Section V.

\section{I/Q IMBALANCE MODEL}

The FD I/Q imbalance of the DCT is modeled as shown in Fig. 1. Although there exists $\mathrm{I} / \mathrm{Q}$ imbalance models in literatures [1], [2], [8] which is based on the equivalent baseband I/Q imbalance, this model directly gives the RF I/Q imbalance model for the convenience of derivation..

In ideal DCT, the complex baseband signal:

$$
x(t)=x_{I}(t)+j \cdot x_{Q}(t)
$$

is modulated by quadrature modulator and the RF signal

$$
\begin{aligned}
y(t) & =\Re\left\{x(t) e^{j 2 \pi f_{c} t}\right\} \\
& =x(t) e^{j 2 \pi f_{c} t}+x^{*}(t) e^{-j 2 \pi f_{c} t}
\end{aligned}
$$

According to (1), the desired baseband signal and its mirror are shifted to RF frequency band $f_{c}$ and $-f_{c}$ separately and there is no overlap between these two frequencies. However, the impairments of real devices may cause problematic overlap.
Generally, the DCT impairments consists of the following factors, as shown in Fig. 1:

- The amplitude difference and phase offset between the quadrature LO branch: $g$ and $\theta$. And the DC offset of the I and Q branch: $O_{I}$ and $O_{Q}$. These factors are generally frequency-independent.

- The baseband gain and phase difference between I and Q branch; and the mixer conversion gain and amplifier gain difference between I and $\mathrm{Q}$ branch. These factors contribute the frequency-dependent effect. Apparently, the baseband impairments can be modeled in RF band by shifting the baseband frequency response to the RF. The impulse response of equivalent RF model of FD impairments is: $h_{I}(t)$ and $h_{Q}(t)$.

The aim of FD I/Q imbalance compensation is to alleviate the overall impact of the above factors.

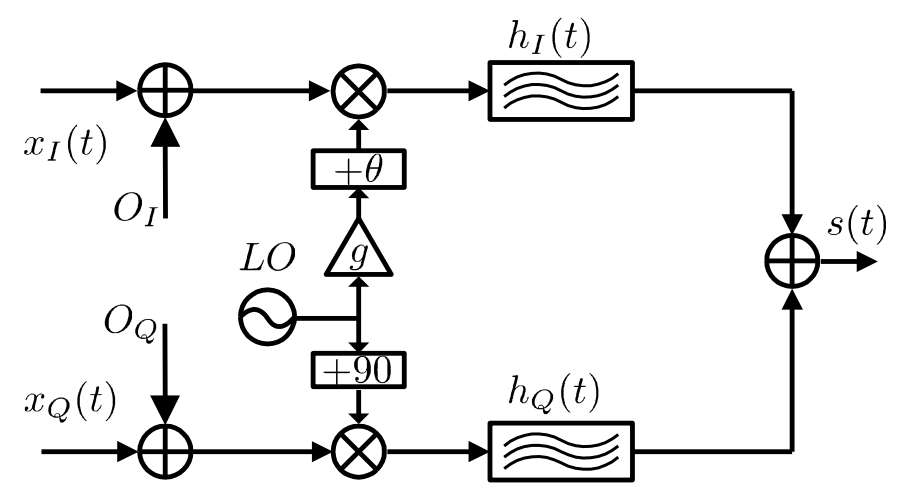

Figure 1. I/Q imbalance model

The corrupted RF signal can be expressed as:

$$
\begin{aligned}
y_{r f}(t)= & \left(x_{I}(t)+O_{I 0}\right) \cdot g \cdot \cos \left(2 \pi f_{c} t+\theta\right) * h_{I}(t)+ \\
& \left(x_{Q}(t)+O_{Q 0}\right) \cdot \cos \left(2 \pi f_{c} t+p i / 2\right) * h_{Q}(t) \\
= & \left(x(t) e^{j 2 \pi f_{c} t}+x^{*}(t) e^{-2 \pi f_{c} t}\right) * g_{1}(t)- \\
& \left(x^{*}(t) e^{j 2 \pi f_{c} t}+x(t) e^{-2 \pi f_{c} t}\right) * g_{2}(t)+ \\
& O(t)
\end{aligned}
$$

where

$$
\begin{aligned}
g_{1}(t) & =\frac{g e^{j \theta} h_{I}(t)+h_{Q}(t)}{2} \\
g_{2}(t) & =\frac{g e^{-j \theta} h_{I}(t)-h_{Q}(t)}{2}
\end{aligned}
$$

and $O(t)$ term is caused by DC offset.

It shows that the desired term $x(t) e^{j 2 \pi f_{c} t}+x^{*}(t) e^{-2 \pi f_{c} t}$ is distorted by the self-distortion term $g_{1}(t)$ and the DC offset term $O(t)$. Apart from that, it is also distorted by $\left(x^{*}(t) e^{j 2 \pi f_{c} t}+x(t) e^{-2 \pi f_{c} t}\right) * g_{2}(t)$ which can be viewed as having resulted from the image signal distorted by $g_{2}(t)$. Notice that $g_{1}(t)$ results in common distortion in I and Q branches, whereas there relative difference between $h_{I}(t)$ and $h_{Q}(t)$, which is related to $g_{2}(t)$ gives rises to I/Q imbalance. The image rejection ratio can now be defined as:

$$
I R R_{d B}(f)=10 \log 10\left(G_{1}^{2}(f) / G_{2}^{2}(f)\right)
$$

The typical IRR of DCT without compensation is around $-25 \sim-40 \mathrm{~dB}$ [1]. 


\section{I/Q IMBALANCE ESTIMATION AND COMPENSATION SCHEME}

In this section, I/Q imbalance estimation and compensation scheme will be discussed. Firstly, the conventional I/Q-demodulation-based method is introduced. Based on this, the new estimation scheme is discussed. Finally, the baseband compensator is introduced.

\section{A. Conventional I/Q-demodulation-based method}

The conventional I/Q-demodulation-based method is shown in Fig. 2.

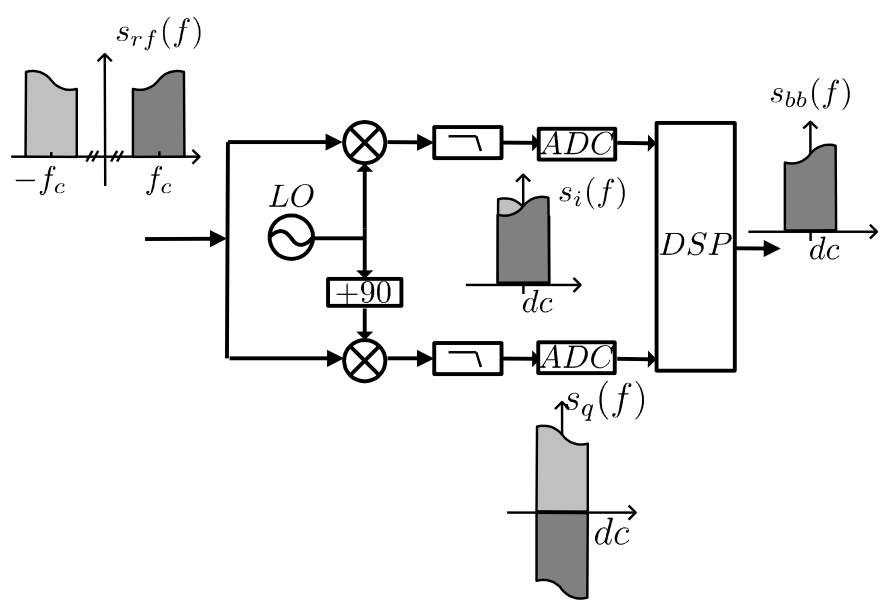

Fig. 2 I/Q-demodulation-based method

As shown in Section I, the accurate estimate of $g, \theta, h_{I}(t)$ and $h_{Q}(t)$ is essential. In I/Q demodulator, the single side band spectrum of RF signal is shifted to the baseband. Hence, the single side band frequency response of $h_{I}(t), h_{Q}(t)$ as well as $g$ and $\theta$ can be estimated. The critical factor in I/Q demodulator is inphase and quadrature LO which separates the inphase and the quadrature components of the RF signal. However, the inherent $\mathrm{I} / \mathrm{Q}$ imbalance and other impairment brings extra estimation error and the impairments is jointly estimated with transmitters in transceiver based schemes [9]-[11].

\section{B. Proposed estimation method}

Enlightened by the conventional I/Q-demodulation-based method, this proposal employs a self-I/Q-demodulation compensation scheme, which omits the external I/Q demodulator to reduce the cost. The self-compensation algorithms in [5]-[7] make use of diode in power detector for frequency-independent I/Q imbalance parameters estimation, namely $g, \theta$. In these schemes, the RF signal is self-demodulated by square law of the diode. However, these diodes are narrow band in generic transmitters. Similarly, the paper proposes wideband diode or even a multiplier to be used for FD I/Q imbalance estimation, as shown in Fig. 3. It obviously required to find out that the self-I/Q-demodulation can only be realized by using the transmitter LO or the transmitted signal itself under the condition that external demodulator is omitted.

Hence, the proposed algorithm utilizes two critical factors: transmitter LO and wideband diode. Based on this, the algorithm is designed so that the signal can be self-demodulated by the non-ideal transmitter quadrature LO pair through the diode. However, the constraint behind this idea is: 1). the transmitter LO is corrupted by these impairments: $g, \theta, O_{I}$ and $O_{Q}$. 2). Transmitting the inphase and quadrature LO simultaneously causes interference to the I and Q demodulation because the inphase and quadrature LO will intermodulate each other in the diode. Therefore, the proposed algorithm consists of the following two steps: 1). Estimate the LO impairments and DC offset estimation: $g, \theta, O_{I}$ and $O_{Q} ; 2$ ). Transmit the training signal including the inphase and quadrature LO by the "time division" method. The demodulation errors caused by LO impairments and DC offset is then compensated. Finally, the overall FD I/Q imbalance parameters are calculated from the self-I/Q-demodulation results. The detailed estimation process is presented below:

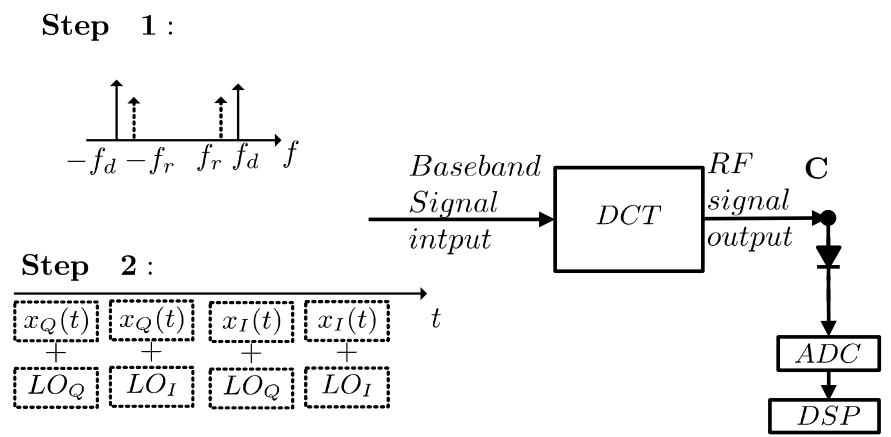

Fig. 3 Proposed Self-IQ-demodulation based compensation scheme

1) LO impairments and DC offset estimation

LO impairments and DC offset estimation are studied in [5]-[7]. [5] proposes a measurement approach, which makes use of the diode in the envelope detector, however it is only suitable for QPSK and Continuous Phase Modulation (CPM) systems. [6] and [7] proposes estimation methods in generic situations. Nonetheless, the computation complexity is relatively high because of the matrix inversion operation when a large number of samples are involved.

In this proposal, the LO impairments $g \theta$ and DC offset $O_{I}$, $O_{Q}$ can be used to estimate at low complexity due to the specially designed test signal. Consider two tones whose frequency $f_{d}$ and $f_{r}$ are close to each other and are passed through diode, as the Step 1, shown in Fig. 3. These two tones intermodulate each other's results in the output which are located at $\pm\left(f_{d}+f_{r}\right)$ and $\pm\left(f_{d}-f_{r}\right)$ respectively. If the frequencies $f_{d}$ and $f_{r}$ are low enough, the imbalance parameter can be viewed as frequency-independent, which means $g, \theta$, $O_{I}$ and $O_{Q}$ can be obtained from the intermodulation products located at $\pm\left(f_{d}+f_{r}\right)$ as described in following steps:

a. Transmit signal $x_{1}(t)=x_{I 1}(t)+j \cdot x_{Q 1}(t)$ where $x_{I 1}(t)=\cos \left(2 \pi f_{d} t\right)+\cos \left(2 \pi f_{r} t\right)$ and $x_{Q 1}(t)=-\sin \left(2 \pi f_{r} t\right)$ in I and Q branch. Omitting the FD effect, then the output signal of the diode can be rewritten as: 
where $k$ is complex gain and

$$
y_{\text {diode }}(t)=k y_{r f}^{2}(t)
$$

$$
\begin{aligned}
y_{r f}(t)= & \Re\left\{\left(O_{I 0}+j O_{Q 0}\right) e^{j 2 \pi f_{c} t}\right. \\
& +\frac{1}{2}\left(g e^{j \theta}+1\right) e^{j 2 \pi\left(f_{c}+f_{r}\right) t}+\frac{1}{2}\left(g e^{j \theta}-1\right) e^{j 2 \pi\left(f_{c}-f_{i}\right.} \\
& \left.+\frac{1}{2} g e^{j \theta}\left(e^{j 2 \pi\left(f_{c}+f_{d}\right) t}+e^{j 2 \pi\left(f_{c}-f_{d}\right) t}\right)\right\} * h
\end{aligned}
$$

Apply FFT to $y_{\text {diode } 1}(t)$, and the complex gain of the tone at frequency $f_{i}=f_{d}-f_{r}$ is:

$$
V_{f_{i} 1}=\left\lceil g^{2}+j g \sin \theta\right\rceil \cdot K e^{j \varphi}
$$

where $K^{j \varphi}$ is the overall gain of transmitter and diode.

b. Similarly, transmit the signal $x_{2}(t)=x_{I 2}(t)+j \cdot x_{Q 2}(t)$ where $x_{I 2}(t)=\cos \left(2 \pi f_{r} t\right)$ and $x_{Q 2}(t)=-\left(\sin \left(2 \pi f_{d} t\right)+\sin \left(2 \pi f_{r} t\right)\right)$, and the complex gain at frequency $f_{i}=f_{d}-f_{r}$ is

$$
V_{f_{i} 2}=[1-j g \sin \theta] \cdot K e^{j \varphi}
$$

c. Solving (5) and (6), we can get:

$$
\begin{gathered}
\hat{g}=\sqrt{\frac{\frac{\Im\left\{V_{f_{i} 1}\right\}}{r r}+\frac{\Re\left\{V_{f_{i}}\right\}}{i i}}{\frac{\Re\left\{V_{f_{i} 2}\right\}}{i i}+\frac{\Im\left\{V_{f_{i} 2}\right\}}{r r}}} \\
\hat{\theta}=\arcsin \left(\frac{\frac{\Im\left\{V_{f_{i} 1}\right\}}{\Im\left\{V_{f_{i}}\right\}}-\frac{\Re\left\{V_{f_{i} 1}\right\}}{\Re\left\{V_{f_{i} 2}\right\}}}{\frac{r r}{\Im\left\{V_{f_{i}}\right\}}+\frac{i i}{\Re\left\{V_{f_{i} 2}\right\}}} \cdot \frac{1}{g_{\text {est }}}\right)
\end{gathered}
$$

where $r r=\Re\left\{V_{f_{i} 1}\right\}+\Re\left\{V_{f_{i}}\right\}$ and $i i=\Im\left\{V_{f_{i} 1}\right\}+\Im\left\{V_{f_{i} 2}\right\}$

d. Estimation of DC offset. Transmit signals $x_{3}(t)=x_{I 3}(t)+j \cdot x_{Q 3}(t)$ where $\quad x_{I 3}(t)=\cos \left(2 \pi f_{d} t\right)$, $x_{Q 3}=0 ; \quad x_{4}(t)=x_{I 4}(t)+j \cdot x_{Q 4}(t)$ where $x_{I 4}=0$, $x_{Q 4}=\sin \left(2 \pi f_{d} t\right)$ and $x_{5}(t)=x_{I 5}(t)+j \cdot x_{Q 5}(t)$ where $x_{I 5}=0, x_{Q 5}=\sin \left(2 \pi f_{d} t\right)+O_{q 5}$ respectively. Assuming that the I and Q branch have been compensated, then the complex gain of signal 3,4 and 5 at frequency $f_{d}$ can be expressed as:

$$
\begin{aligned}
V_{f_{d} 3} & =\left(g^{2} O_{I 0}+g \sin \theta O_{Q 0}\right) \cdot G \\
V_{f_{d} 4} & =-j\left(g \sin \theta O_{I 0}+O_{Q 0}\right) \cdot G \\
V_{f_{d} 5} & =-j\left(g \sin \theta O_{I 0}+O_{Q 0}+O_{Q 5}\right) \cdot G
\end{aligned}
$$

where

$$
\hat{O}_{I 0}=\frac{V_{f_{d} 3}-\hat{g} \sin (\hat{\theta}) V_{f_{d} 4}}{\hat{g}^{2}\left(1-\sin ^{2}(\hat{\theta})\right) \hat{G}}
$$

$$
\begin{aligned}
& \hat{O}_{Q 0}=\frac{\hat{g} V_{f d 4}-\sin \hat{\theta} V_{f d 3}}{\hat{g}\left(1-\sin ^{2}(\hat{\theta})\right) \hat{G}} \\
& \hat{G}=\frac{j\left(V_{f_{d} 5}-V_{f_{d} 4}\right)}{O_{Q 5}}
\end{aligned}
$$

$i=3,4,5 ; G$ is the path gain.

2) FD related I/Q impairments parameters estimation

This step will give the estimate of $H_{I}(f)$ and $H_{Q}(f)$. As presented earlier, the FD I/Q imbalance parameters can be estimated by using I/Q demodulator. In [2], RF signal is demodulated in digital domain by a pair of LO which are strictly orthogonal. This method requires a high speed ADC that digitizes the IF signal. The estimators in [9]-[11] adopts zeros-IF structure, which estimates the impairment of transmitter and receiver jointly.

This paper achieves I/Q demodulation by mixing RF signal and transmitter LO in the diode as shown in Fig. 3. The interference arises if the inphase and quadrature LO are transmitted simultaneously. Hence, if the single I or Q branch signal $s(t)$ along with the inphase or quadrature LO is transmitted at different time, the self-I/Q-demodulation of single I or Q branch signal can be achieved with the help of square law of the diode. The demodulation error caused by LO impairments and DC offset can be compensated using the estimation results in Step 1. This "time division" method is introduced below:

a. Inphase demodulation of signal from I branch.

Transmit signal $\quad x_{6}(t)=x_{I 6}(t)+j \cdot x_{Q 6}(t) \quad$ where $x_{I 6}(t)=s(t)+s^{*}(t)-\hat{O}_{I 0}+O_{I 6}$ and $x_{Q 6}(t)=-\hat{O}_{Q 0}$ in the first signal period $t \subseteq(0, T)$. (2) can be rewritten as:

$$
y_{r f}(t)=\left(s(t)+s^{*}(t)+O_{I 6}\right) \cdot g \cos \left(2 \pi f_{c} t \theta\right) * h_{I}(t)
$$

The frequency domain signal at point $\mathrm{C}$ in Fig. 3 is:

$$
\begin{aligned}
Y_{\text {diode6 }}(f)= & F F T\left\{k y_{r f}^{2}(t)\right\} \\
= & g^{2}\left(S(f)+S^{*}(-f)\right) \\
& \cdot\left(H_{I}\left(f-f_{c}\right)+H_{I}\left(f+f_{c}\right)\right) \cdot K \\
& +I_{s}(f)+I_{l o}(f)
\end{aligned}
$$

where $K$ is the overall complex gain; $I_{s}(f)$ is the inter-modulation product of term $s(t)+s^{*}(t) ; I_{l o}(f)$ is the inter-modulation product of LO itself.

As shown in (16), the first term is the interested signal, which indicates that the RF frequency response of I branch is shifted to baseband by the LO from I branch. However, the diode inter-modulation also produces $I_{s}(t)$ and $I_{l o}(t)$. For $I_{l o}(t)$, its frequency is located at $2 f_{c}$ because of the square law. As to $I_{s}(t)$, it is comprised of the square output of $s(t)+s^{*}(t)$. Hence, the frequency band of $I_{s}(t)$ and interested signal can be separated if $s(t)$ is carefully designed. Based on this assumption, the following derivation will focus on the interested baseband signal and omit the last two terms in (16). 
Denote the baseband equivalent of $H_{I}\left(f-f_{c}\right)$ as $\tilde{H}_{I}(f)$. It is easy to prove that the baseband equivalent of $H_{I}\left(f+f_{c}\right)$ is $\tilde{H}_{I}^{*}(-f)$. Similarly, the baseband equivalent of $H_{Q}\left(f-f_{c}\right)$ and $H_{Q}\left(f+f_{c}\right)$ are denoted as $\tilde{H}_{Q}(f)$ and $\tilde{H}_{Q}^{*}(-f)$ respectively. Then (16) can be rewritten as:

$$
\begin{aligned}
Y_{\text {diode6 }}(f)= & g^{2}\left(S(f)+S^{*}(-f)\right) \\
& \cdot\left(\tilde{H}_{I}(f)+\tilde{H}_{I}^{*}(-f)\right) \cdot K
\end{aligned}
$$

b. Quadrature demodulation of signal from Q branch.

Transmit signal $\quad x_{7}(t)=x_{I 7}(t)+j \cdot x_{Q 7}(t) \quad$ where $x_{I 7}(t)=s(t-T)+s^{*}(t-T)-\hat{O}_{I 0}$ and $x_{Q 7}(t)=-\hat{O}_{Q 0}+O_{Q 7}$ in the second signal period $t \subseteq(T, 2 T]$. The frequency domain signal at point $\mathrm{C}$ in Fig. 3 is:

$$
\begin{aligned}
Y_{\text {dioder }}(f)= & j\left(S(f)+S^{*}(-f)\right) \\
& \cdot\left(g e^{-j \theta} \tilde{H}_{I}(f)-g e^{j \theta} \tilde{H}_{I}^{*}(-f)\right) \cdot K
\end{aligned}
$$

c. Inphase demodulation of signal from I branch.

Transmit signal $x_{8}(t)=x_{I 8}(t)+j \cdot x_{Q 8}(t)$ where $x_{I 8}(t)=-\hat{O}_{I 0}+O_{I 8}$ and $x_{Q 8}(t)=-j\left(s(t-2 T)-s^{*}(t-2 T)\right)-\hat{O}_{Q 0}$ in the third signal period $t \subseteq(2 T, 3 T$. The frequency domain signal at point $\mathrm{C}$ in Fig. 3 is:

$$
\begin{aligned}
Y_{\text {diode8 }}(t)= & \left(S(f)-S^{*}(-f)\right) \\
& \cdot\left(g e^{-j \theta} \tilde{H}_{Q}^{*}(-f)+g e^{j \theta} \tilde{H}_{Q}(f)\right) \cdot K
\end{aligned}
$$

d. Quadrature demodulation of signal from $\mathrm{Q}$ branch.

Transmit signal $\quad x_{9}(t)=x_{I 9}(t)+j \cdot x_{Q 9}(t) \quad$ where $x_{I 9}(t)=-\hat{O}_{I 0}$ and

$x_{Q 9}(t)=-j\left(s(t-3 T)-s^{*}(t-3 T)\right)-\hat{O}_{Q 0}+\hat{O}_{Q 9}$ in the fourth signal period $t \subseteq(3 T, 4 T)$. The frequency domain signal at point $\mathrm{C}$ in Fig. 3 is:

$$
\begin{aligned}
Y_{\text {diode } 9}(f)= & j\left(-S(f)+S^{*}(-f)\right) \\
& \cdot\left(\tilde{H}_{Q}^{*}(-f)+\tilde{H}_{Q}(f)\right) \cdot K
\end{aligned}
$$

Solve (17)-(19) by using estimation results in (7) (8), the estimation of baseband equivalent FD parameters are:

$$
\begin{aligned}
\hat{H}_{I}(f) & =\frac{Y_{\text {diode6 }}(f)-j \hat{g} e^{-j \hat{\theta}} Y_{\text {diode7 }}(f)}{\hat{g}^{2}\left(1+e^{-2 j \hat{\theta}}\right)\left(X(f)+X^{*}(-f)\right) K} \\
\hat{H}_{Q}(f) & =\frac{Y_{\text {diode8 }}(f)-j \hat{g} e^{-j \hat{\theta}} Y_{\text {diode }}(f)}{\hat{g}\left(e^{j \hat{\theta}}+e^{-j \hat{\theta}}\right)\left(X^{*}(-f)-X(f)\right) K}
\end{aligned}
$$

The overall complex gain $K$ is still unknown. However, it is not necessarily required if the compensator is carefully designed. The design details are presented as follows.
Apparently, the proposed algorithm utilizes the same oscilloscope as the transmitter in order to avoid the frequency errors. The proposed method is based on the assumption that the square law device works on in ideal conditions. In the real world scenario, the $3^{\text {rd }}$ and other higher order inter-modutation terms produce interference. Therefore, it overlaps LO and the interested signal, which actually jeopardizes the overall estimation performance. Hence, the simplified training signal $x(t)$ with only one tone produces the least interference and yields most accurate estimation results.

The whole estimation process flow is summarized as:

1. Transmit signal $x_{1}(t) \sim x_{5}(t)$ and estimate LO impairment $\hat{g}, \hat{\theta}$ and DC offset $\hat{O}_{I 0}, \hat{O}_{Q 0}$.
2. Transmit signal $x_{6}(t) \sim x_{9}(t)$ sequently and calculate the FD related I/Q imbalance parameters: $H_{I}(f), H_{Q}(f)$

Fig. 4 Self-I/Q-demodulation based Estimation Process

\section{I/Q imbalance compensation}

After the parameters are estimated, the I/Q imbalance can be compensated. This paper adopts the baseband compensation scheme in [1]:

$$
x_{p}(t)=x(t)+w(t) * x^{*}(t)
$$

where $w(t)$ is the compensator impulse response, as shown in Fig. 6.

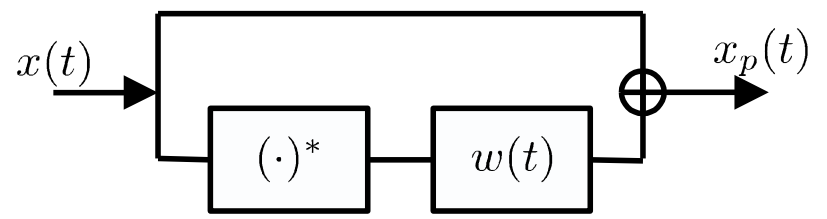

Fig. 6 Transmitter I/Q imbalance compensator

The optimum baseband compensator $w(t)$ that minimizes the mirror component in (2) is given by [1]:

$$
W_{\text {opt }}(f)=-\tilde{G}_{2}(t) / \tilde{G}_{1}(f)
$$

where

$$
\begin{aligned}
\tilde{G}_{1}(f) & =\frac{\hat{g} e^{j \tilde{\theta}} \hat{H}_{I}(f)+\hat{H}_{Q}(f)}{2} \\
\tilde{G}_{2}(f) & =\frac{\hat{g} e^{-j \hat{\theta}} \tilde{H}_{I}(f)-\tilde{H}_{Q}(f)}{2}
\end{aligned}
$$


According to (24), the common complex gain $K$ in (21) and (22) is cancelled and will not contribute to the compensated signal.

\section{SimULATION AND LABORATORY RESULTS}

In this section, the I/Q imbalance compensation performance is investigated by computer simulation and laboratory measurements. A laboratory test platform is set up to evaluate the achievable performance as shown in Fig. 6. The target compensation DCT is the Aeroflex PXI 3320/3050 arbitrary waveform generator. Its bandwidth can be $80 \mathrm{MHz}$ with typical IRR about -35 - $40 \mathrm{~dB}$. As the passband of target DCT diode is $0-2 \mathrm{Mhz}$, the estimation process for wideband I/Q imbalance parameters is emulated using digitized RF output of PXI 3320/3050: The output RF signal is directly digitized by a Tektronix oscilloscope 6804B at sampling rate of 10 GSPS. Then the digitized RF signal is uploaded to a PC. The PC simulates the diode process and estimates the FD I/Q imbalance using the proposed algorithm. The Aeroflex spectrum analyzer is used to observe the IRR performance Note that the LO impairments $g, \theta$ and DC offset $O_{I}, O_{Q}$ is estimated using the internal diode as it can meet the narrow band estimation requirements.

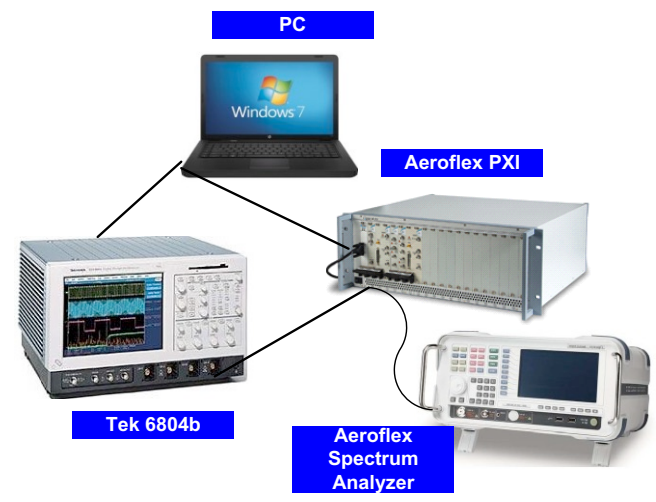

Fig. 6 Test Platform

The estimation performance Step 1 is presented in Fig. 7 and Table 1. This Step aims to estimate LO impairments $g, \theta$ and DC offset $O_{I}, O_{Q}$. Since these parameters are frequencyindependent, the narrow band test tones $f_{d}=20 \mathrm{KHz}$ and $f_{r}=19 \mathrm{KHz}$ are used to verify the estimation performance in Step 1. According to Fig. 7, the estimation algorithm yields nearly $80 \mathrm{~dB}$ IRR which proves the effectiveness of the algorithm in Step 1. Table 1 also listed the achievable performance for different bandwidths. According to the table, the IRR decreases as the bandwidth is increasing, which indicates that the frequency-dependent factor has large impact as bandwidth is increasing.

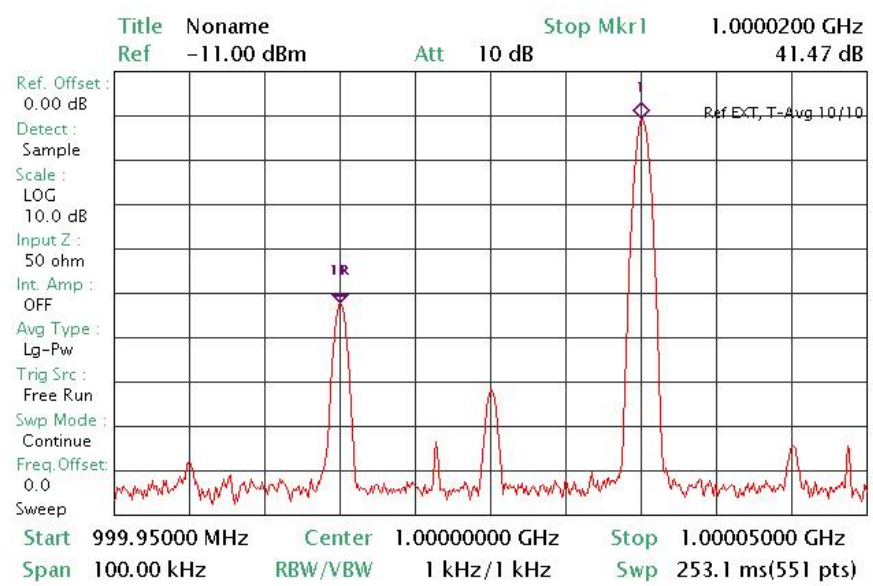

Fig.7 (a) $20 \mathrm{KHz}$ upper band signal before frequency-independent I/Q imbalance compensation

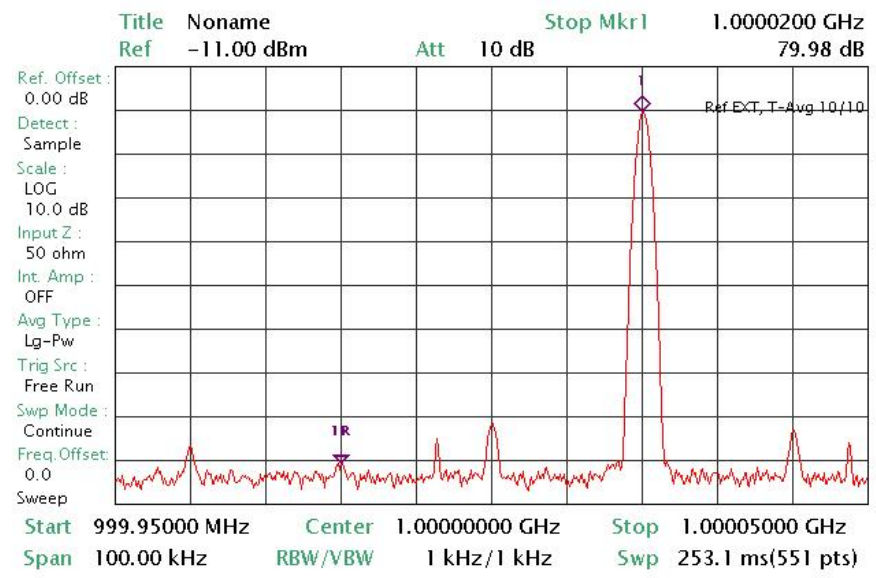

Fig.7 (a) $20 \mathrm{KHz}$ upper band signal after frequency-independent I/Q imbalance compensation

Table 1: Measured IRR before and after compensation for different $f_{d}$ and $L O$ in $d B$

\begin{tabular}{|c|c|c|c|c|}
\hline & \multicolumn{2}{|c|}{2} & \multicolumn{2}{c|}{4} \\
\hline & Before & After & Before & After \\
\hline 500 & 34.12 & 70.42 & 38.15 & 69.59 \\
\hline 1000 & 33.47 & 64.79 & 38.32 & 67.75 \\
\hline 5000 & 34.78 & 50.12 & 38.58 & 52.27 \\
\hline
\end{tabular}

The wideband FD I/Q imbalance compensation performance is evaluated in Fig.8. Fig. 8 shows the IRR performance of the proposed method at frequency $f_{d}=75 \mathrm{MHz}$. In this experiment, the estimation results of Step 1 are utilized to compensate the demodulation errors caused by LO impairments $g, \theta$ and DC offset $O_{I}, O_{Q}$. The directly digitized RF signal is utilized for FD parameters estimation. After the parameters are estimated, both the upper and lower band signals $x(t)=e^{j 2 \pi f_{d} t}$ and $x(t)=e^{-j 2 \pi f_{d} t}$ are generated to verify the estimation and compensation performance on both sidebands. It shows that the proposed estimation algorithm gives more that $50 \mathrm{~dB}$ IRR on both sidebands which indicates the overall FD I/Q imbalance in wideband is compensated well. 


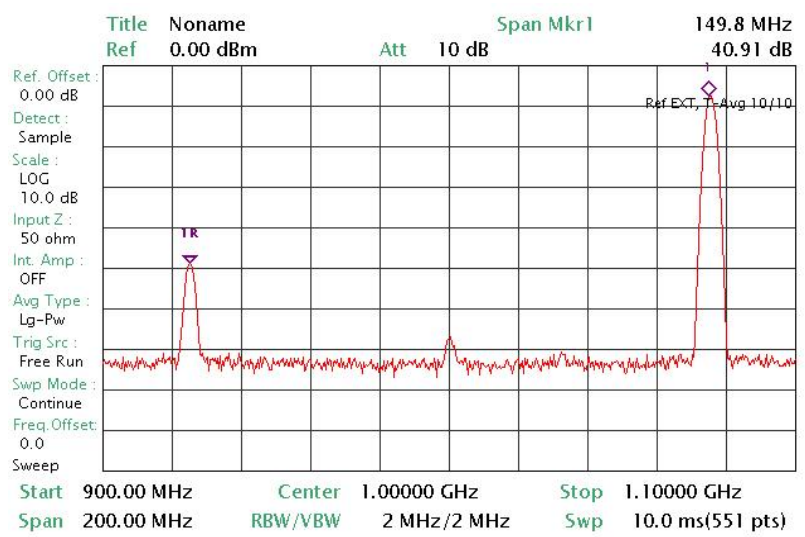

(a)

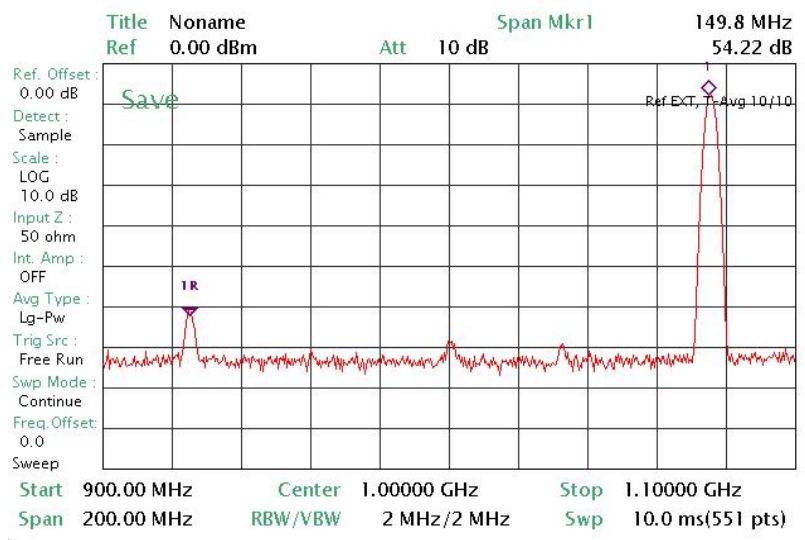

(c) compensation; (d) Lower band signal after compensation

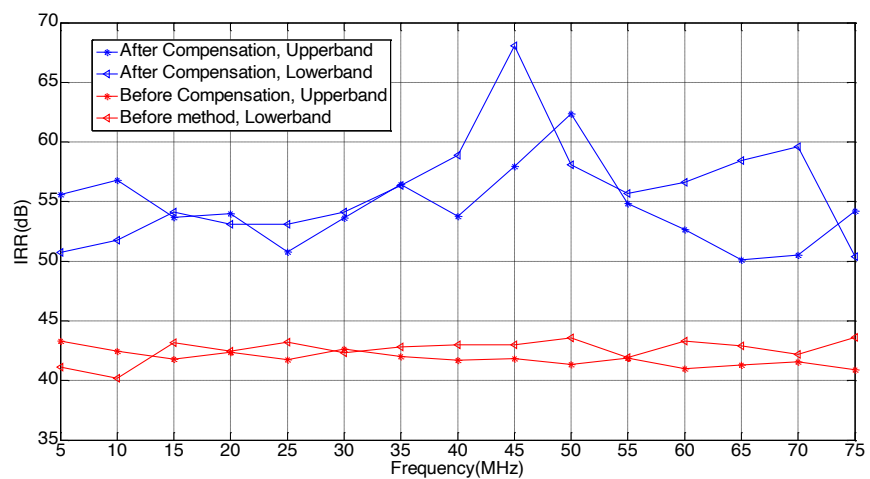

Fig. 9 Compensation performance

Fig. 9 shows the IRR performance of the proposed method within the whole signal bandwidth. In this experiment, the estimation results in Step 1 are used. In Step 2, single tone signal $x(t)=e^{-j 2 \pi f_{d} t}$ is utilized for estimation. According to this figure, the compensation scheme gives around $10 \mathrm{~dB}$ IRR enhancement within the whole signal bandwidth.

According to (7), (8), (12), (13), (21) and (22), the computation complexity of proposed algorithm is relatively low which requires 6 FFT operations and a few multiplication and addition operations, as compared to the computation complexity of the conventional algorithm, which requires

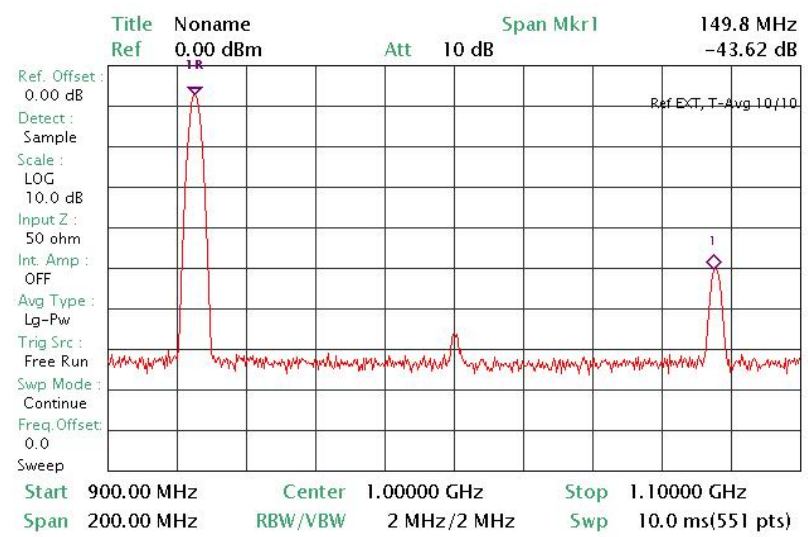

(b)

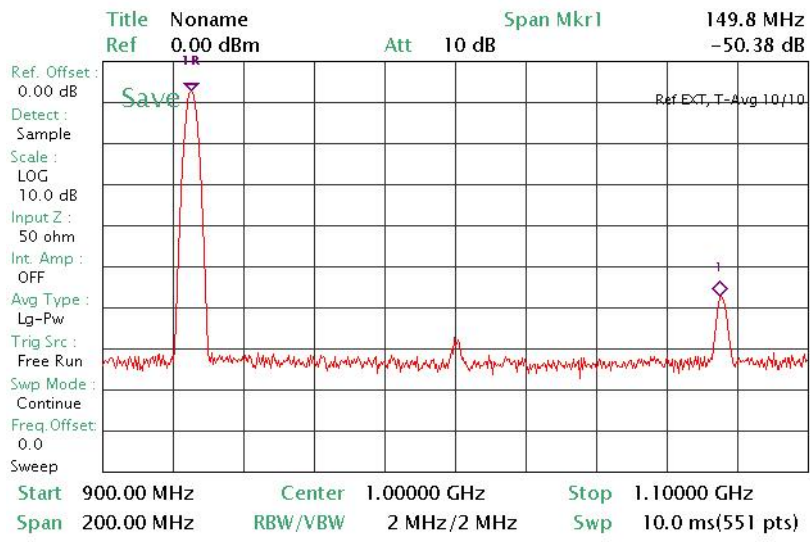

(d)

matrix inverse operations is $O\left(n^{3}\right)$. As to the cost, this proposed algorithm only requires a wideband diode and ADC, which is more cost effective than the conventional method, which uses external/internal modulator or even instruments.

\section{CONCLUSIONS}

This paper proposes self-I/Q-demodulation based compensation scheme of FD I/Q imbalance without using external estimation instruments. Based on the existing narrow band compensation scheme, minor modification will need to be made on the internal power detection circuit to estimate FD I/Q imbalance parameters. The LO impairments $g, \theta$ and DC offset $O_{I}, O_{Q}$ are firstly estimated. Based on these results, the estimator makes use of transmitter LO and realizes self-I/Qdemodulation by "time division" method. Afterwards, the FD parameters are estimated. The corresponding compensation scheme are also presented. The performance is verified by laboratory measurements, which reveals the effectiveness of the compensation scheme. The computation complexity is relatively low and the implementation cost is low as only a wideband diode and ADC is required compared with the conventional scheme that requires an external demodulator and high speed ADCs. 


\section{REFERENCES}

[1] L. Anttila, M. Valkama, M. Renfors, "Frequency-Selective IQ Mismatch Calibration of Wideband Direct-Conversion Transmitters" IEEE Trans on Vehicular Technology, vol. 57, pp 2099-2113, July 2008

[2] L. Ding, Z. Ma, D.R. Morgan, M. Zierdt, "Frequency dependent modulator imbalance in predistortion linearization systems: Modeling and compensation" in Proc. Asilomar Conf. Signals, Syst., Comp., Pacific Grove, CA, Nov. 2003, pp. 688-692.

[3] K. Sun, I. Darwazeh, L. Huang, A. Jones, "Optimal pilot based FD I-Q imbalance compensation for wideband direct-conversion transmitters", $A$ in Proc. WCNC, 2012. Paris, France, Apr. 1-4, 2012, pp. 226-231

[4] I. Lu, J. Chang, "Joint Transmitter and Receiver IQ Imbalance Estimation and Compensation for OFDM Systems" in Proc. of IEEE International Conference on Radio and Wireless Symposium (RWS), pp. 476-479, New Orleans, LA, Jan. 2010.

[5] M. Faulkner, T. Mattsson, and W. Yates, "Automatic adjustment of quadrature modulators," Electron. Letters, vol. 27, pp. 214-216, Jan. 1991.

[6] X. Huang and M. Caron, "Efficient transmitter self-calibration and amplifier linearization techniques," in Proc. IEEE Int. Circuits Syst. Symp., May 2007, New Orleans, LA, pp. 265-268.

[7] S. Burglechner, G. Hueber, A, Springer, "On the Estimation and Compensation of IQ Impairments in Direct Conversion Transmitters", Proc. Euro. Conf. Wireless Tech., Oct. 27-28, 2008, Amsterdam, Holland, pp. 69-72.

[8] M. Kim, Y. Maruichi, J. Takada "Parametric Method of FD I/Q Imbalance Compensation for Wideband Quadrature Modulator" IEEE Tranl. on Microwave Theory and Techniques., vol. 61, pp. 270-280. Jan. 2013
[9] A. Nassery, S. Ozev, "An analytical technique for characterization of transceiver IQ imbalances in the loop-back mode," in Design, Automation \& Test in Europe Conference \& Exhibition (DATE),Mar. 2012, pp.1084-1089.

[10] C. Zhang, Z, Xiao, B. Gao, L. Su, "Three-Stage Treatment of TX/RX IQ Imbalance and Channel with CFO for SC-FDE Systems" IEEE Communication Letters, vol. 18, pp. 297-300, Feb. 2014.

[11] M. Marey, M. Samir, O.A. Dobre, "EM-Based Joint Channel Estimation and IQ Imbalances for OFDM Systems", IEEE Trans. On Broadcasting, vol. 58, pp. 106-113, March 2012.

[12] A. Prochazka, R. Neumann, "High frequency distortion analysis of a semiconductor diode for CATV applications", IEEE Trans. on Consumer Electronics, Vol CE-21, No. 2, May 1975

[13] M. Rawat, F.M. Ghannouchi, "A Mutual Distortion and Impairment Compensator for Wideband Direct-Conversion Transmitters Using Neural Networks", IEEE Trans. on Broadcasting, Vol. 58, pp. 168-177, Apr. 2014.

[14] P. Garcia, A. Ortega, J. de Mingo, A. Valdovinos, "Nonlinear distortion cancellation using LINC transmitters in OFDM systems", IEEE Trans. on Broadcasting, vol. 51, pp. 84-93, March, 2005

[15] Z. Zheng, Z. Yang, C. Pan,Y. Zhu, "Effects of nonlinear distortion and imperfect parameters estimation on the performance of OFDM-based DTTB systems", IEEE Trans. on Broadcasting. vol. 51. pp. 237- 243. Jun. 2005

[16] A. Chini, Yiyan. Wu, M. EI-Tanany, S. Mahmoud. "Hardware nonlinearities in digital TV broadcasting using OFDM modulation", IEEE Trans. on Broadcasting. vol. 44. pp. 12- 21. Aug. 2012 